\title{
Diversité Et Abondance Des Micromammifères Terrestres: Indicateurs De L'anthropisation De La Forêt Classée De Monogaga, Sud-Ouest De La Côte d'Ivoire
}

\author{
Akpatou Kouamé Bertin \\ Laboratoire de Zoologie et Biologie Animale, UFR Biosciences, \\ Université Félix Houphouët-Boigny, Abidjan, Côte d'Ivoire

\section{Bohoussou Kouakou Hilaire} \\ UFR Ingénierie Agronomique Forestière et Environnementale, \\ Université de Man, Côte d'Ivoire
}

\section{Kadjo Blaise}

Laboratoire de Zoologie et Biologie Animale, UFR Biosciences,

Université Félix Houphouët-Boigny, Abidjan, Côte d'Ivoire

\section{N'goran Kouakou Eliezer}

Laboratoire de Zoologie et Biologie Animale, UFR Biosciences,

Université Félix Houphouët-Boigny, Abidjan Côte d'Ivoire

\section{Adou Yao Constant Yves}

Laboratoire de Botanique, UFR Biosciences, Université Félix Houphouët-

Boigny, Abidjan Côte d'Ivoire

Doi: 10.19044/esj.2018.v14n36p52 URL:http://dx.doi.org/10.19044/esj.2018.v14n36p52

\begin{abstract}
Anthropization of Classified Forests $(\mathrm{CF})$ is a recurrent phenomenon in Côte d'Ivoire. It particularly affects coastal forests including Monogaga classified forest. Currently, flora composition and vegetation structure are the only biological tools available to better appreciate impacts of anthropic pressures on Monogaga classified forest. Yet, fauna and more particularly terrestrial small mammals constitute an essential component of this forest. Thus, this study aims to contribute to the understanding of the effects of anthropization of Monogaga classified forest based on "terrestrial small mammal" model. We used conventional Sherman traps to fairly investigate terrestrial small mammal communities in four plots (A, B, C and D) subjected to different anthropogenic pressures through Monogaga classified forest. Plots A and B were more disturbed than plots C and D. We collected 66 specimens in 1920 trap nights. These specimens are distributed among seven rodent species Dephomys defua, Hybomys trivirgatus, Hylomyscus simus,
\end{abstract}


Lophuromys sikapusi, Malacomys edwardsi, Mastomys erythroleucus and Praomys rostratus and one soricomorph Crocidura olivieri. Abundances of terrestrial small mammals are higher in plots $C$ and D. In the same way, diversity indices decrease from the most conserved to the least conserved plots. However, differences in species richness are weak between plots. Hierarchical classification of the four plots based on small mammal's assemblages allows a good segregation. This study confirms that "terrestrial small mammal" model can contribute to the assessment of anthropization of forest habitats in lower Côte d'Ivoire.

Keywords: Small mammals, Diversity, Abundance, Anthropization, Monogaga Classified Forest

\section{Résumé}

L'anthropisation des Forêts Classées (FC) est récurrente en Côte d'Ivoire. Elle touche particulièrement les forêts côtières dont la FC Monogaga. Seules la flore et la structure de la végétation permettent d'apprécier les impacts des pressions anthropiques dans la FC Monogaga. Pourtant la faune et plus particulièrement les micromammifères terrestres constituent une composante essentielle de cette forêt. Cette étude se base sur le modèle «micromammifère terrestre» pour contribuer à la compréhension de l'anthropisation de la FC Monogaga. Ainsi, quatre parcelles (A, B, C et D) soumises à des pressions anthropiques différentes ont été équitablement prospectées à l'aide de pièges conventionnels de type Sherman. Les parcelles A et B étant plus perturbées que les parcelles C et D. Un effort global de piégeage estimé à 1920 nuits-pièges à permis de collecter 66 individus répartis entre sept espèces de rongeurs Dephomys defua, Hybomys trivirgatus, Hylomyscus simus, Lophuromys sikapusi, Malacomys edwardsi, Mastomys erythroleucus et Praomys rostratus et une espèce de soricomorphes Crocidura olivieri. Les abondances de micromammifères terrestres sont plus élevées dans les parcelles $\mathrm{C}$ et $\mathrm{D}$. Il en est de même pour les indices de diversités qui décroissent des parcelles les plus conservées vers les moins conservées. Cependant, les richesses spécifiques diffèrent faiblement entre les parcelles. La classification hiérarchique basée sur les niveaux d'assemblage des communautés de micromammifères terrestres permet de bien ségréguer les parcelles. Cette étude confirme que le modèle «micromammifère terrestre » peut contribuer à l'appréciation du niveau d'anthropisation des habitats forestiers en basse Côte d'Ivoire.

Mots-clés: Micromammifères, Diversité, Abondance, Anthropisation, Forêt classée de Monogaga 


\section{Introduction}

Les forêts ivoiriennes sont soumises à de fortes pressions anthropiques depuis plusieurs décennies (Bakayoko et al., 2004). Les forêts dans le sudouest de la Côte d'Ivoire ont véritablement connu la fragmentation après l'avènement de la route côtière construite pour faciliter l'accès à de nombreuses villes balnéaires et au port de San-Pédro (Adou Yao, 2005). Ainsi, la forêt classée de Monogaga (FCM), située à mi-chemin sur la côtière va particulièrement attirer les exploitants forestiers et les planteurs de caféiers et de cacaoyers. Face à ces pressions anthropiques, la Société pour le Développement des Forêts (SODEFOR), en sa qualité de gestionnaire officiel des forêts en Côte d'Ivoire, a élaboré un plan d'aménagement basé sur le zonage en deux séries dont une dite «Série agricole » dans laquelle les paysans sont autorisés à cultiver et l'autre dite «Série de protection », exclusivement réservée à la conservation et à la valorisation des ressources floristiques et fauniques (Adou Yao et al., 2011). Cependant, les besoins croissants de populations riveraines n'ont pas permis de respecter le zonage de la forêt classée de Monogaga tel que conçu par la SEDEFOR. Cette situation va se traduire par de nombreuses intrusions dans la série de protection. Adou Yao et al. (2011) révèlent que l'impact des intrusions dans la série de protection est plus perceptible au niveau de la structure de la végétation que de la diversité floristique. Seule la structure des formations végétales permet de mieux apprécier les différences entre la gestion adoptée par la SODEFOR et les pratiques agricoles des paysans (Adou Yao et al., 2011). En effet, les compositions spécifiques des différentes formations végétales étudiées n'ont pas permis d'établir une nette ségrégation des habitats soumis à ces deux différents modes de gestion de la forêt classée de Monogaga.

L'appréciation des différents modes de gestion de la forêt classée de Monogaga souffre encore de la nécessité de la prise en compte d'un modèle biologique autre que la flore. A ce jour, aucun modèle faunique n'a été étudié afin de comprendre l'impact des différents modes de gestion de cette forêt. Pourtant, de nombreux travaux admettent qu'une bonne évaluation des stratégies de gestion forestière doit tenir compte des différentes composantes qui caractérisent l'écosystème forestier (Chatelain et al., 2010 ; Edgar, 2010 ; Leroy et al., 2013 ; Koné et al., 2014 ; Balineau et al., 2017).Toutefois, il a été démontré ailleurs, que les différents niveaux de perturbation des milieux naturels peuvent être appréciés sur la base des modifications observées dans leurs compositions fauniques originelles (Hamrick, 2007 ; Bogdziewicz \& Zwolak, 2013 ; Gentili et al., 2014 ;). Cette assertion est plus vraisemblable 
avec les espèces animales dont les réponses aux perturbations de leurs milieux naturels sont perceptibles (Stork et al., 2009; Schwitzer et al., 2011; Buitrago-Suarez et al., 2015 ).

Les communautés de micromammifères terrestres présentent pour leur part, plusieurs paramètres biologiques pouvant varier en fonction des niveaux de perturbation de leur environnement. En effet, la diversité et l'abondance des micromammifères peuvent être considérées comme des paramètres écologiques capables d'aider à l'appréciation de l'empreinte écologique des activités humaines dans les milieux naturels (Men et al., 2015). Mistrot (2000) a montré que l'exploration des communautés de micromammifères peut non seulement renseigner sur les paléoclimats, mais également sur l'histoire récente de l'anthropisation des milieux.

Cette étude est une contribution à la compréhension de l'impact des actions anthropiques dans la forêt classée de Monogaga. Elle se base sur le modèle «micromammifère terrestre» et prend en compte les variations de la richesse spécifique, de la diversité et de l'abondance des espèces pour comprendre les effets de l'anthropisation de la forêt classée de Monogaga.

\section{Matériel et méthodes}

\section{Site de l'étude}

La Forêt Classée de Monogaga (FCM) est située dans le sud ouest de la Côte d'Ivoire, entre $4^{\circ} 46^{\prime}$ et $4^{\circ} 58^{\prime}$ de latitude nord et $6^{\circ} 15^{\prime}$ 'et $6^{\circ} 35^{\prime}$ de longitude ouest. Elle est limitée à l'ouest par la rivière Brimé, à l'est par une limite conventionnelle d'environ $17 \mathrm{Km}$ de plantation de Gmelina arborea Roxb, au nord par la route côtière et au sud par l'Océan Atlantique, (figure 1). La FCM fait partie des principales formations forestières situées le long du littoral ivoirien. Le relief de la FCM n'excède pas 130 mètres d'altitude, ce qui lui vaut le qualificatif de forêt de basse altitude (Chatelain \& Piguet, 1999). Elle couvre une superficie de 40000 ha et présente la particularité d'avoir été classée avec des populations humaines vivant à l'intérieur (arrêté n66/SER/DAM, du 30 juin 1973; Adou Yao et al., 2011). La FCM est caractérisée par plusieurs types de végétations dont les plus importantes sont les forêts à Eremospatha macrocarpa (Man et Wendl.) Wendl et Diospyros mannii Hiern, les forêts à Diospyros spp. et Mapania spp., sur terre ferme dans les interfluves, des forêts marécageuses sur sols hydromorphes et des formations littorales (Aké-Assi, 1984 ; 1997). Guillaumet \& Adjanohoun (1971) classent la FCM parmi les forêts denses humides sempervirentes.

\section{Description des parcelles de piégeage}

Quatre parcelles (A, B, C et D) ont été prospectées dans le cadre de cette étude (figure 1). Chaque parcelle présente une végétation et une flore 
relativement homogène et bénéficie d'un statut particulier en fonction de son histoire et/ou du type de gestion auquel elle est soumise (Adou Yao et al., 2011). La parcelle A est située dans de la série de protection et bénéficie des actions de conservation conduites par la SODEFOR. Elle est très proche du village de Moussadougou reconnu comme étant le village le plus peuplé des localités environnantes de la FCM. De ce fait, les paysans s'y rendent régulièrement pour exploiter différentes ressources afin de satisfaire à leurs besoins quotidiens. La parcelle B est proche du littoral. Il s'agit d'une ancienne cocoteraie abandonnée depuis plus de 25 ans. Elle est également incluse dans la série de protection mais jouit des actions de protection intégrale. Les activités des paysans dans cette vieille jachère se résument en la collecte d'escargots géants Achatina fulica. La parcelle C se situe également dans la série de protection, toutefois elle bénéficie d'une protection particulière vu la composition exceptionnelle de sa flore et son attrait touristique. Les visites touristiques et les patrouilles des forestiers sont les principales formes de présence humaine dans cette zone relativement mieux conservée. La parcelle $\mathrm{D}$ est un site d'exploitation forestière, abandonné depuis plus d'une soixantaine d'années. Elle est située dans la série de protection et essentiellement soumise aux activités de braconnage en plus des activités de patrouille des forestiers de la SODEFOR.

\section{Piégeage des micromammifères terrestres, identification et autopsie des spécimens capturés}

Les différentes parcelles ont été prospectées à l'aide de dispositifs de piégeage installés sur des superficies de 0,56 ha $(70 \mathrm{~m}$ x $80 \mathrm{~m})$. La végétation de chacune des quatre parcelles a été caractérisée selon Adou Yao et al. (2011) en vue de ressortir les différences structurales entre elles. Cette étude s'est focalisée sur les Muridés et les Soricidés. Ainsi, le dispositif de piégeage a été simplifié comparativement à ceux employés par Dosso (1983) et Wilson et al. (1996). Quatre lignes de piégeage de $80 \mathrm{~m}$ de long chacune ont été disposées parallèlement à équidistance de $20 \mathrm{~m}$. Quinze postes de piégeage ont été marqués sur chaque ligne à équidistance de $5 \mathrm{~m}$ entre les postes consécutifs. Les postes de piégeage extrêmes étant à cinq mètres de l'extrémité de chaque ligne de piégeage. A chaque poste de piégeage, deux pièges conventionnels de type Sherman $(7,5 \times 9 \times 23 \mathrm{~cm})$ ont été placés chacun à l'extrémité d'une ligne imaginaire perpendiculaire à la ligne de piégeage. Les deux pièges du même poste de piégeage sont distants de 10 mètres, soit cinq mètres de part et d'autre de la ligne de piégeage. Chaque campagne de piégeage a duré quatre nuits consécutives, soit un effort de piégeage estimé à 1920 nuits-pièges pour l'ensemble des quatre parcelles. Les pièges ont été appâtés à l'aide de mésocarpe de graine de palmier à huile (Elaeis guineensis). Toutes les parcelles ont été inventoriées à la même période en vue de minimiser l'effet 
des variations saisonnières sur les abondances des micromammifères terrestres. Les animaux capturés ont été collectés, autopsiés et identifiés sur le site de capture. Cette première identification des spécimens a été confirmée par une seconde, après comparaison à des spécimens muséologiques de référence du Muséum National d'Histoire Naturelle de Paris (MNHN) en France et du Musée Royal de l'Afrique Centrale (MRAC) de Tervuren en Belgique. En cas d'incertitude après ces deux étapes d'identification, les spécimens concernés ont été inclus dans des analyses phylogénétiques moléculaires au MNHN, en vue de clarifier leur statut taxinomique. Les espèces ont été identifiées en suivant la nomenclature de Happold (2013) pour les rongeurs et de Happold \& Happold (2013) pour les soricomorphes.

\section{Mesure de la richesse et de la diversité biologique des parcelles}

Les espèces collectées lors des campagnes de piégeage sont enregistrées dans une base de données conçue à cet effet à l'aide du logiciel Excel. Les richesses spécifiques $(\mathrm{S})$ des parcelles sont extraites de cette base de données. La diversité biologique des parcelles a été estimée en utilisant l'indice de Shannon (1948) libellée ci-dessous:

$$
H^{\prime}=-\sum \text { pi } \log _{2} \text { pi, avec pi }=(\mathrm{ni} / \mathrm{N})
$$

où ni et $\mathrm{N}$ désignent respectivement le nombre d'individus d'une espèce dans l'échantillon et le nombre total d'individus de toutes les espèces dans ce même l'échantillon. Le choix de cet indice s'explique par le fait qu'il prend en compte à la fois l'abondance et la richesse spécifique.

L'appréciation de la régularité des espèces dans les différentes parcelles a été faite à partir du calcul de l'indice d'équitabilité (E). Cet indice désigne le rapport entre la diversité observée $\left(\mathrm{H}^{\prime}\right)$ et la diversité théorique maximale du peuplement (Hmax). Il est exprimé comme suit :

$\mathrm{E}=\mathrm{H}^{\prime} / \mathrm{Hmax}=\mathrm{H}^{\prime} / \log _{2} \mathrm{~S}$, avec $\mathrm{Hmax}=\log _{2} \mathrm{~S}$ où $\mathrm{S}$ est la richesse spécifique. Le traitement des données a été fait à l'aide de la version $7.5 \mathrm{du}$ logiciel EstimateS (Colwell, 2004).

\section{Ressemblance et regroupement des parcelles de piégeage}

Le regroupement des parcelles de piégeage s'est fait sur la base des abondances et des co-occurrences des espèces de micromammifères. Les données originales ont été préalablement réduites en deux matrices de synthèse ou tableaux de contingence. Les deux matrices ont été soumises au test d'indépendance (G2) en vue de s'assurer de la relation entre les communautés de micromammifères et les différents types de parcelles. La première matrice qui prend en compte toutes les espèces (rares et abondantes) a été utilisée pour le calcul des similarités entre les paires de parcelles. La mesure de la similarité a été faite à l'aide du coefficient de Bray \& Curtis (1957) suivant la formule: 


$$
\mathrm{Cz}=2 \mathrm{w} / \mathrm{a}+\mathrm{b}
$$

où $\mathrm{w}$ est la somme des valeurs d'abondance les plus faibles pour chaque espèce commune aux deux parcelles $\mathrm{A}$ et $\mathrm{B}$; a est la somme des abondances de toutes les espèces trouvées dans la parcelle $\mathrm{A}$; et $\mathrm{b}$ est la somme des abondances de toutes les espèces trouvées dans la parcelle $\mathrm{B}$. Ce coefficient fait partie du groupe de coefficients de similarité utilisant les données quantitatives et excluant le double zéro. Selon Legendre \& Legendre (1984), l'exclusion du double zéro se justifie par le fait que l'absence d'une espèce de deux parcelles ne peut être interprétée comme une indication de ressemblance. Dans la seconde matrice, toutes les espèces rares ont été exclues de l'analyse en vue de ne regrouper les parcelles que sur la base des espèces les plus régulières. Cette matrice a été utilisée pour l'analyse factorielle des correspondances qui a permis de regrouper des parcelles suivant la composition de la communauté de faune murine et soricine. L'estimation des similarités a été faite à l'aide de la version 7.5 du logiciel EstimateS (Colwell, 2004).

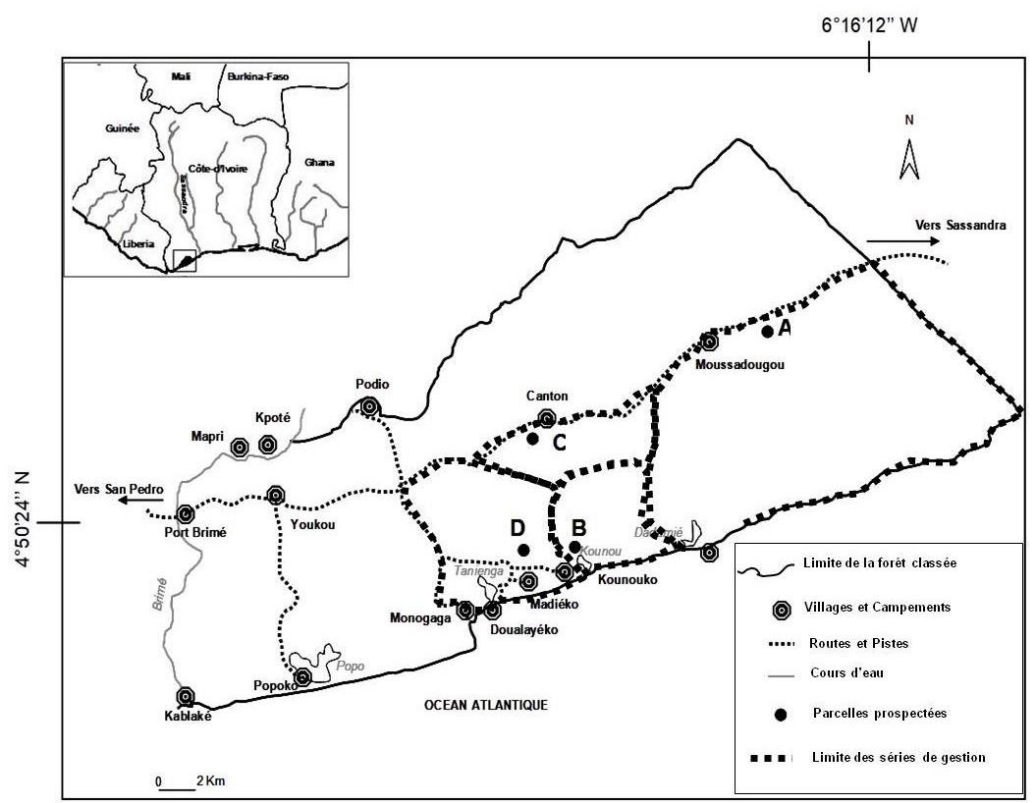

Figure 1 : Répartition géographique des parcelles de piégeage de micromammifères dans la forêt classée de Monogaga, Côte d'Ivoire.

\section{Résultats}

\section{Occurrence et abondance des micromammifères terrestres}

Un total de 66 individus de micromammifères terrestres a été obtenu en 1920 nuits-pièges, ce qui donne un succès de piégeage de 3,44 \%. Ces individus appartiennent à deux familles (Soricidés et Muridés) et sont répartis entre huit espèces. La famille des Muridés est la plus abondante avec 57 
spécimens répartis entre sept genres et sept espèces (Tableau 1). Celle des Soricidés est représentée par un seul genre avec l'espèce Crocidura olivieri. Les espèces les plus abondantes de Muridés sont Malacomys edwardsi ( $\mathrm{n}=$ 18), Hylomyscus simus $(\mathrm{n}=14)$, Praomys rostratus $(\mathrm{n}=13)$. Crocidura olivieri (Soricidés) et Lophuromys sikapusi sont moyennement représentées avec respectivement neuf et huit spécimens. Trois espèces, Mastomys erythroleucus $(\mathrm{n}=2)$, Hybomys trivirgatus $(\mathrm{n}=1)$ et Dephomys defua $(\mathrm{n}=1)$ sont très faiblement représentées.

L'analyse parcellaire montre que dans la parcelle A, la communauté de micromammifères terrestres est dominée par Praomys rostratus $(\mathrm{n}=5)$ ce qui représente 38,46\% de l'ensemble des captures. L'espèce Hylomyscus simus $(\mathrm{n}=1)$ a été la moins capturée dans cette parcelle (Tableau 1). Dans la celle B, trois espèces Crocidura olivieri, Hylomyscus simus et Praomys rostratus co-dominent avec trois individus chacune (Tableau 1). Malacomys edwardsi ( $n=9)$ est dominante dans la parcelle C, suivie de Praomys rostratus $(\mathrm{n}=4)$. Les espèces Crocidura olivieri et Hylomyscus simus ont été les moins collectées dans la parcelle C (Tableau 1). Dans la parcelle D, Hylomyscus simus est la plus abondante $(\mathrm{n}=8)$. La seconde espèce dominante est Malacomys edwardsi $(\mathrm{n}=7)$. Les espèces Dephomys defua, Hylomyscus simus et Praomys rostratus $(\mathrm{n}=1)$ enregistrent les plus faibles captures dans la parcelle D (Tableau 1).

Praomys rostratus, Hylomyscus simus et Crocidura olivieri sont largement distribuées dans la forêt classée de Monogaga comparativement aux autres espèces. Ces trois espèces ont été saisies dans toutes les parcelles prospectées (Tableau 1). Par contre, Dephomys defua, Hybomys trivirgatus et Mastomys erythroleucus n'ont été capturées que dans la parcelle D. Malacomys edwardsi a été prise dans toutes les parcelles sauf la parcelle B.

\section{Richesse spécifique et diversité des micromammifères terrestres}

Les piégeages dans les quatre parcelles prospectées ont permis de recenser huit espèces de micromammifères terrestres. La parcelle $\mathrm{D}$ présente la richesse spécifique la plus élevée $(S=7)$. Elle est suivie des parcelles A et $\mathrm{C}$ qui enregistrent le même nombre d'espèces $(\mathrm{S}=5)$. La parcelle $\mathrm{B}$ présente la plus faible richesse spécifique $(\mathrm{S}=4)$, (Tableau 1). La valeur la plus élevée des indices de diversité de Shannon a été observée dans la parcelle $\mathrm{D}\left(\mathrm{H}^{\prime}=\right.$ 1,84). Les parcelles $\mathrm{C}$ et $\mathrm{B}$ ont enregistré des valeurs respectives de $\mathrm{H}^{\prime}=1,82$ et $\mathrm{H}^{\prime}=1,80$. La plus faible valeur a été obtenue dans la parcelle $\mathrm{A}\left(\mathrm{H}^{\prime}=1,64\right)$, (Tableau 1).

Les indices d'équitabilité (E) sont élevés dans les quatre parcelles échantillonnées (Tableau 1). Toutefois la plus forte valeur a été relevée dans la parcelle $\mathrm{B}(\mathrm{E}=0,99)$. La parcelle $\mathrm{D}(\mathrm{E}=0,82)$ a enregistré la faible valeur, (Tableau 1). 
Tableau 1. Abondance et diversité des micromammifères terrestres dans quatre parcelles différentes de la forêt classée de Monogaga.

\begin{tabular}{lllll|l}
\hline \multirow{2}{*}{ Espèce } & \multicolumn{3}{l|}{ Parcelle de piégeage } & Total \\
\cline { 2 - 7 } & $\mathrm{A}$ & $\mathrm{B}$ & $\mathrm{C}$ & $\mathrm{D}$ & \\
\hline Crocidura olivieri (Lesson, 1827) & 2 & 3 & 2 & 2 & 9 \\
Dephomys defua (Miller, 1900) & - & - & - & 1 & 1 \\
Hybomys trivirgatus (Temminck, 1853) & - & - & - & 1 & 1 \\
Hylomyscus simus (Aellen \& Coolidge, 1930) & 1 & 3 & 2 & 8 & 14 \\
Lophuromys sikapusi (Temminck, 1853) & 3 & 2 & 3 & - & 8 \\
Malacomys edwardsi Rochebrune, 1885 & 2 & - & 9 & 7 & 18 \\
Mastomys erythroleucus (Temminck, 1853) & - & - & - & 2 & 2 \\
Praomys rostratus (Miller, 1900) & 5 & 3 & 4 & 1 & 13 \\
\hline Nombre d'individus & 13 & 11 & 20 & 22 & 66 \\
Nombre d'espèces (S) & 5 & 4 & 5 & 7 & 8 \\
Indice de Shanonn-Wiener $\left(H^{\prime}\right.$ ) & 1,64 & 1,80 & 1,82 & 1,84 & \\
Indice d'Equitabilité (E) & 0,92 & 0,99 & 0,88 & 0,82 & \\
\hline
\end{tabular}

Similarité entre les quatre parcelles selon les communautés de micromammifères terrestres

Les coefficients de similarité entre les quatre parcelles prises deux à deux varient entre 0,34 et 0,73 (Tableau 2). Le binôme constitué par les parcelles $\mathrm{A}$ et $\mathrm{C}$ présente le coefficient de similarité le plus élevé $(0,73)$. Ces deux parcelles ont cinq espèces en commun sur les huit espèces collectées dans cette étude. Les coefficients de similarité les plus faibles $(0,34)$ et $(0,36)$ sont obtenus avec les couples de parcelles (A, D) et (B, D) respectivement. Seulement trois espèces sont communes aux parcelles au sein de ces deux couples (Tableau 2).

Tableau 2. Coefficients de similarité entre les quatre parcelles selon les communautés de micromammifères terrestres.

\begin{tabular}{|c|c|c|c|}
\hline Parcelles & Nombre d'espèces & Espèces communes & Coefficient de similarité \\
\hline $\mathrm{A}$ & 6 & & \multirow{2}{*}{0,67} \\
\hline $\mathrm{B}$ & 7 & 4 & \\
\hline A & 6 & & \multirow{2}{*}{0,73} \\
\hline$\overline{\mathrm{C}}$ & 6 & 5 & \\
\hline A & 6 & & \multirow{2}{*}{0,34} \\
\hline $\bar{D}$ & 6 & 4 & \\
\hline $\mathrm{B}$ & 7 & & \multirow{2}{*}{0,58} \\
\hline $\mathrm{C}$ & 6 & & \\
\hline B & 7 & & \multirow{2}{*}{0,36} \\
\hline $\bar{D}$ & 6 & & \\
\hline $\mathrm{C}$ & 6 & & \multirow{2}{*}{0,57} \\
\hline $\bar{D}$ & 6 & & \\
\hline
\end{tabular}




\section{Classification hiérarchique des parcelles de piégeage selon les assemblages de micromammifères terrestres}

La classification des parcelles sur la base des assemblages des espèces de micromammifères terrestres les plus régulières permet de définir trois groupes. Le premier groupe est représenté par deux entités que sont les parcelles $\mathrm{A}$ et $\mathrm{B}$. Le deuxième et le troisième groupe sont respectivement représentés par les parcelles $\mathrm{C}$ et $\mathrm{D}$ (figure 2). La classification appliquée à la représentativité numérique des espèces de micromammifères terrestres a permis de dresser la liste de celles qui caractérisent le mieux chaque parcelle ou groupe de parcelles. Ainsi, le groupe formé par les parcelles A et B est plus marqué par six espèces. Lophuromys sikapusi, Crocidura olivieri et Praomys rostratus caractérisent mieux pour la parcelle A, tandis que la parcelle B se détermine prioritairement par Mastomys erythroleucus, Dephomys defua et Hybomys trivirgatus. Hylomyscus simus et Malacomys edwardsi sont numériquement plus marquantes dans la ségrégation des parcelles $\mathrm{C}$ et $\mathrm{D}$ respectivement, tableau 2 (figure 2).
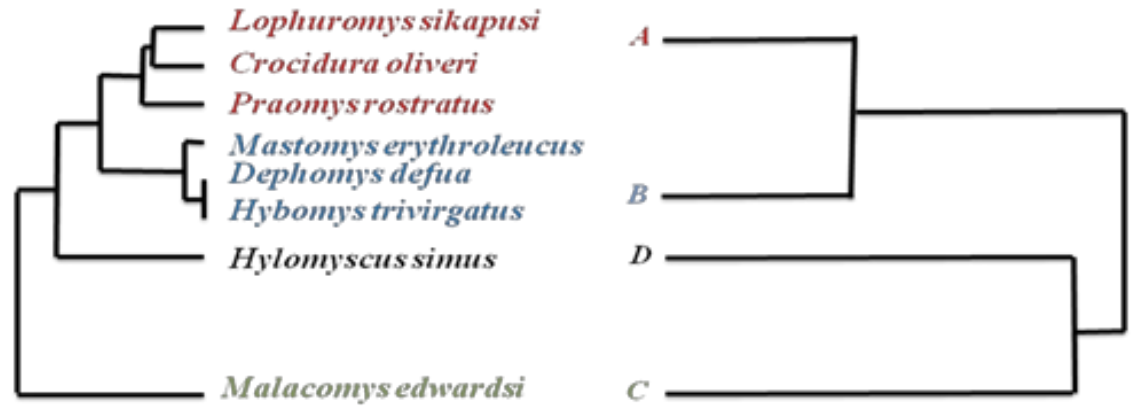

Figure 2 : Classification hiérarchique des parcelles de piégeage avec la présentation des assemblages de micromammifères terrestres associés.

\section{Discussion}

La richesse spécifique obtenue dans cette étude est de huit espèces de micromammifères terrestres sur l'ensemble des quatre parcelles prospectées. Elle est faible comparativement à Kadjo et al. (2013) qui en dénombrent treize au Parc National du Banco qui appartient au même domaine écologique que la forêt classée de Monogaga. Par ailleurs, Adam (1977) et Dosso (1983) signalent la présence d'une vingtaine de micromammifères terrestres au niveau des forêts littorales de la Côte d'Ivoire. Seule une espèce de Crocidure est signalée dans cette étude. Pourtant, le genre Crocidura regorge d'au moins dix espèces à travers les reliques de forêts situées au sud de la Côte d'Ivoire y compris la forêt classée de Monogaga (Jacquet et al., 2014). La faible richesse spécifique globalement constatée dans cette étude serait due entre autres à l'insuffisance de l'effort de piégeage. Cependant, cette étude signale la présence des principales espèces de micromammifères terrestres des forêts 
côtières ivoiriennes en comparaison aux travaux de Heim de Balsac \& Aellen (1965) et de Dosso (1983). La parcelle D présente la richesse spécifique la plus élevée. Adou Yao et al. (2011) signalent que cette parcelle est soumise aux activités combinées de la SODEFOR et des paysans, créant ainsi une variété d'habitats dégradés. Cette variété d'habitats expliquerait sa plus forte richesse en espèces de micromammifères comparativement aux parcelles $\mathrm{A}, \mathrm{B}$ et $\mathrm{C}$. En effet, la communauté de micromammifères terrestres de la parcelle D renferme les principales espèces forestières que sont Malacomys edwardsi et Hylomyscus simus, de même que les espèces des milieux ouverts représentées par Hybomys trivirgatus, Dephomys defua et Mastomys erythroleucus. La présence de Dephomys defua et de Hybomys trivirgatus dans la parcelle D est un indicateur des actions anthropiques et de diverses perturbations naturelles antérieures survenues dans ce milieu. Dephomys defua et Hybomys trivirgatus étant indicatrices respectivement des trouées ou de chablis et des habitats ouverts (Decher, 1997; Happold, 2013). La diversité des microhabitats dans la parcelle D soulignée par Adou yao et al. (2011) pourrait offrir des conditions favorables à l'établissement de plusieurs espèces de micromammifères terrestres (Magige \& Senzota, 2006 ; Fichet-Calvet et al., 2009 ; Makundi et al., 2009). La capture de Mastomys erythroleucus dans la parcelle D est une marque de l'anthropisation. En effet, Adou Yao et al. (2011) signalent la présence d'espèces végétales caractéristiques des jachères, ce qui confirme les activités champêtres conduites dans cette parcelle quelques années plus tôt. Ces anciens champs auraient favorisé l'établissement de Mastomys erythroleucus qui selon Duplantier \& Granjon (1988) préfère les champs et les milieux perturbés. La capture de Praomys rostratus, Hylomyscus simus et de Crocidura olivieri dans tous les habitats prospectés, témoigne de leur plus grande capacité d'adaptation aux modifications du milieu forestier comme signalé par Happold (2013).

Les parcelles $\mathrm{C}$ et $\mathrm{D}$ présentent les abondances les plus élevées du fait des espèces typiquement forestières que sont Malacomys edwardsi et Hylomyscus simus. Ces deux espèces sont faiblement représentées dans les parcelles A et B qui sont les plus perturbées. Ce résultat atteste que les habitats forestiers des parcelles C et D offrent encore les conditions favorables au développement de Malacomys edwardsi et Hylomyscus simus. Ce constat de la variation de l'abondance des micromammifères selon le niveau de perturbation de leur habitat naturel a déjà été signalé par Dosso (1983) et Decher (1997).

Les indices de diversité calculés sont proches de ceux obtenus par Kadjo et al. (2013) au Parc National du Banco. Ces indices décroissent des milieux les moins perturbés vers les milieux les plus perturbés. Dans cette étude, les parcelles $\mathrm{C}$ et $\mathrm{D}$ qui sont les moins perturbées, présentent les indices de diversité les plus élevés. La longue période de mise en jachère de la parcelle 
$\mathrm{D}$ et la conservation particulière de la parcelle $\mathrm{C}$ ont certainement permis l'établissement des principales espèces forestières que sont Malacomys edwardsi et Hylomyscus simus. Ces deux espèces sont les plus abondantes dans les parcelles $\mathrm{C}$ et $\mathrm{D}$ d'où les indices de diversité les plus élevés. Ce constat est soutenu par Gentili et al. (2014). Ces auteurs ont montré que l'indice de diversité spécifique évolue inversement à la perturbation du milieu par les activités humaines. Ceci serait dû à l'abondance élevée des espèces spécialistes, tel est le cas ici, de Malacomys edwardsi et Hylomyscus simus.

Les quatre parcelles prospectées dans la forêt classée de Monogaga sont ségréguées en trois groupes sur la base de la spécificité des assemblages des espèces de micromammifères terrestres. Chaque groupe ainsi constitué se caractérise par la spécificité de la communauté de micromammifères en réponse au degré d'anthropisation. En effet, les parcelles $\mathrm{C}$ et $\mathrm{D}$ qui présentent les plus faibles niveaux d'anthropisation se démarquent des parcelles A et B du fait de l'abondance élevée de Malacomys edwardsi. Selon Rosevear (1969), soutenu par Happold (2013) et Akpatou et al. (2018), Malacomys edwardsi est une espèce typiquement forestière et serait très sensible aux perturbations de 1'habitat forestier. La classification hiérarchique des parcelles montre pourtant que la parcelle $\mathrm{D}$ se démarque de la parcelle $\mathrm{C}$ avec laquelle les abondances de Malacomys edwardsi sont concomitamment élevées. Cette démarcation se justifierait par l'abondance plus élevée du Hylomyscus simus dans la parcelle D. Selon Adou yao et al. (2011), la parcelle D est caractérisée par un sous bois relativement clairsemée. Happold (2013) mentionne que ce type d'habitat est propice à l'établissement des espèces du genre Hylomyscus. En effet, Hylomyscus simus tolère mieux les perturbations du milieu forestier comparativement à Malacomys edwardsi (Dosso, 1983 ; Happold, 2013). Les parcelles A et B dont les habitats sont plus exposés aux actions anthropiques (Adou Yao et al., 2011), forment un seul groupe dont les communautés de micromammifères sont essentiellement représentées par Praomys rostratus, Crocidura olivieri et Lophuromys sikapusi. Ces espèces sont connues pour leur plus grande capacité d'adaptation aux perturbations du milieu forestier (Dosso, 1983 ; Akpatou, 2009 ; Happold, 2013; Houémènou et al., 2014). L'absence de capture de Hybomys trivirgatus, Dephomys defua et Mastomys erythroleucus dans les parcelles A B et C s'expliquerait par le fait qu'elles aient échappé au dispositif de piégeage plutôt qu'à une absence dans ces sites.

\section{Conclusion}

Les différentes formes d'anthropisation de la forêt classée de Monogaga n'ont pas profondément modifié les richesses spécifiques des communautés de micromammifères terrestres. Cependant, les abondances des espèces typiquement forestières telles que Malacomys edwardsi et Hylomyscus simus décroissent fortement des milieux les plus conservés vers les milieux les moins conservés. Il en est de même pour les indices de diversités calculés pour 
chacune des quatre parcelles prospectées. Ainsi, l'abondance des micromammifères combinée à la diversité spécifique des milieux peuvent être utilisées pour apprécier les niveaux d'anthropisation dans la forêt classée de Monogaga. Cette étude montre également que l'anthropisation de la forêt classée de Monogaga peut être constatée et appréciée par la présence de Dephomys defua, Mastomys erythroleucus et Hybomys trivirgatus qui sont des espèces caractéristiques des milieux ouverts. En conséquence, les micromammifères terrestres constituent un bon modèle biologique pour l'évaluation de l'anthropisation des milieux forestiers en basse Côte d'Ivoire.

\section{Remerciements}

Cette étude a été conduite grâce à un financement du Centre Suisse de Recherches Scientifiques en Côte d'Ivoire (CSRS) à qui nous adressons nos remerciements pour la logistique mise à notre disposition. Nous remercions également le Centre for Tropical Research (CTR) de l'Université de Californie à Los Angeles (UCLA) qui a offert gracieusement les pièges et contribué au financement des missions de piégeage. Nous tenons à exprimer nos sincères gratitudes au Muséum National d'Histoire Naturelle de Paris (MNHN) en France et au Musée Royal de l'Afrique Centrale (MRAC) de Tervuren en Belgique pour nous avoir accordés l'accès à leur collection de micromammifères. Enfin, nous sommes reconnaissants à la Direction Générale de la SODEFOR qui nous a accordé l'accès à la forêt classée de Monogaga et autorisé le piégeage des micromammifères terrestres. Nous n'oublions pas les guides locaux pour leur aide lors de cette étude.

\section{References:}

1. Adam, F. (1977). Données préliminaires sur l'habitat et la stratification des rongeurs en forêt de Basse Côte d'Ivoire. Mammalia, 41 : 283-290.

2. Adou Yao, C. Y. (2005). Pratiques paysannes et dynamiques de la biodiversité dans la forêt classée de Monogaga (Côte d' Ivoire). Thèse Doctorat unique, Département Hommes Natures et Société, Université MNHN, Paris, France, 233 p.

3. Adou Yao, C. Y., Bakayoko A., Akpatou K. B., \& N'Guessan K. (2011). Impacts de pressions anthropiques sur la flore et la structure de la végétation dans la forêt classée de Monogaga, Côte d'Ivoire. Journal of Animal \& Plant Sciences, 12 (2) : 1560-1572.

4. Aké-Assi, L. (1984). Flore de la Côte d'Ivoire : étude descriptive et biogéographique avec quelques notes ethnobotaniques. Thèse d'Etat Université d'Abidjan (Côte d'Ivoire). Tome III : 1069- 1206.

5. Aké-Assi, L. (1997). Inventaire floristique de quelques forêts classées de la région côtière sud-ouest de la Côte d'Ivoire : Port Gauthier, Dassioko, Monogaga. Sodefor- Minagra-Union Européenne, 209 p. 
6. Akpatou, K. B., Bohoussou, K. H., Kadjo, B., \& Nicolas, V. (2018). Terrestrial small mammal diversity and abundance in Taï National Park, Côte d'Ivoire. Nature Conservation Research, 3 (Suppl.2), DOI: 10.24189/ncr.2018.067.

7. Akpatou, K. B. (2009). Systématique et phylogéographie des espèces du genre Praomys Thomas 1915 (Rodentia, Muridae) des forêts de la Haute Guinée. Thèse unique d'Université de Cocody, Abidjan, Côte d'Ivoire, UFR Biosciences, 136 p.

8. Bakayoko, A., Martin, P., Gautier, L., Chatelain, C., Traré, D. \& Spichiger, R. (2004). Etude comparative des massifs forestiers entourant la zone de Taï à Zagné (sud-ouest de la Côte d'Ivoire). Candollea 59, 191-229 p.

9. Balineau, G., Bernath, S., \& Pahuatini, V. (2017). Cocoa farmers' agricultural practices and livelihoods in Côte d'Ivoire. Technical reports $\mathrm{N}^{\circ} 24,43 \mathrm{p}$.

10. Bogdziewicz, M., \& Zwolak, R. (2013). Responses of small mammals to clear-cutting in temperate and boreal forests of Europe: a metaanalysis and review. European Journal of Forests Resources, 11p.

11. Bray, R. J., \& Curtis J. T. (1957). An ordination of the upland forest communities of southern Wisconsin. Ecological Monograph, 27: 325349.

12. Buitrago-Suarez, U. A., Jose, I., Mojica, J. I., \& Bonneau, L. K. (2015). Habitat perturbation and survival strategies of the Andean catfish Astroblepus mariae (Fowler, 1919). Revista de la Academia Colombiana de Ciencias Exactas, Físicas y Naturales, 39 (150) : 3641.

13. Chatelain, C., \& Piguet, F. (1999). Evolution du couvert végétal des forêts côtières de Côte d'Ivoire entre 1989-90, 1996 et 1999. Projet Côtière Sodefor-FED. CD-Rom.

14. Chatelain, C., Bakayoko, A., Martin P., \& Gautier, L. (2010) Monitoring tropical forest fragmentation in the Zagne-Tai area (west of Tai National Park, Cote d'Ivoire). Biodiversity and Conservation. 19: 2405-2420.

15. Colwell, R. K. (2004). ESTIMATES: Statistical Estimation of Species Richness and Shared Species from Samples, Version 7.5. Available at http://viceroy.eeb.uconn.edu/ estimates. Persistent URL http://purl.oclc.org/estimates.

16. Decher, J. (1997). Consevation, small mammals, and the future of sacred groves in West Africa. Biodiversity and Conservation, 6: 10071026.

17. Dosso, H. (1983). Etude des Rongeurs de forêts hygrophiles conservées et des zones anthropisées de la Côte d'Ivoire méridionale. 
Thèse de d'Université de Cocody, Abidjan, Côte d'Ivoire, UFR Biosciences, $249 \mathrm{p}$.

18. Duplantier, J. M. \& Granjon, L. (1988). Occupation et utilisation de l'espace par des populations du genre Mastomys au Sénégal : étude de trois niveaux de perception. Sciences et Techniques des Animaux de Laboratoire, 13 : 129-131.

19. Edgar, P., Foster, J., \& Baker, J. (2010). Reptile Habitat Management Handbook. Amphibian and Reptile Conservation, Bournemouth, 84 p.

20. Fichet-Calvet, E., Audenaert, L., Barriere, P., \& Verheyen, E. (2009). Diversity, dynamics and reproduction in a community of small mammals in Upper Guinea, with emphasis on pygmy mice ecology. African Journal of Ecology, 48: 600-614.

21. Gentili, S., Sigura, M., \& Bonesi, L. (2014). Decreased small mammals species diversity and increased population abundance along a gradient of agricultural intensification. Italian-journal-ofmammalogy, 25 (1): 39-44.

22. Guillaumet, J. L., \& Adjanohoun, E. (1971). La végétation naturelle de la Côte d'Ivoire, In : J. M. Avenard, M. Eldin, G. Girard, J. Sir Coulin, P. Touchebeuf, et A. Perraud, (Eds.), Le milieu naturel de la Côte d'Ivoire. Mémoire ORSTOM. 50 : 161-261.

23. Hamrick, W. W. (2007). Small Mammal Habitat Associations in a Fragmented Agricultural Landscape. Electronic Theses \& Dissertations, $103 \mathrm{p}$.

24. Happold, D. C. D., (2013). Mammals of Africa, Volume III : Rodents, Hares and Rabbits. Bloomsbury Publishing, London, $784 \mathrm{p}$.

25. Happold M. \& and Happold DCD: 2013. Mammals of Africa. Volume IV: Hedgehogs, Shrews and Bats. Bloomsbury Publishing, London, United Kingdom, 800 pp.

26. Heim de Balsac H., \& Aellen Aellen, V. (1965). Les Muridae de basse Côte d'Ivoire. Revue Suisse de Zoologie, 72: 695-753.

27. Houémènou, G, Kassa, B., \& Libois, R. (2014). Ecologie, diversité spécifique et abondance des petits mammifères de la ville de Cotonou au Bénin (Afrique de l'Ouest). International Journal of Biological and Chemical Sciences, 8 (3): 1202-1213.

28. Jacquet, F., Nicolas, V., Colyn, M., Kadjo, B., Hutterer, R., Akpatou, B., Cruaud, C., \& Denys, C. (2014). Forest refugia and riverine barriers promote diversification in the West African pygmy shrew (Crocidura obscurior complex, Soricomorpha). Zoologica Scripta, 43: 131-148.

29. Kadjo, B., Kouadio, R. Y., Vogel, V., Dubey, S., \& Vogel, P. (2013). Assessment of terrestrial small mammals and a record of the critically endangered shrew Crocidura wimmeri in Banco National Park (Côte d'Ivoire). Mammalia, 77 (4): 439-446. 
30. Koné, M., Kouadio, Y. L., Danho F. R., Neuba, D. F. R., Malan, D. F., \& Coulibaly, L. (2014). Évolution de la couverture forestière de la Côte d'Ivoire des années 1960 au début du 21e siècle. International Journal of Innovation and Applied Studies, 7 (2): 782-794.

31. Legendre, L., \& Legendre, P. (1984). Ecologie numérique. Tome 2 : La structure des données écologiques. Masson, Presses de l'Université du Québec. 334 p.

32. Leroy, M., Derroire, G., Vendé, J., \& Leménager, T. (2013). La gestion durable des forêts tropicales: De l'analyse critique du concept à l'évaluation environnementale des dispositifs de gestion. Agence Française de Développement, $240 \mathrm{p}$.

33. Magige, F., \& Senzota, R. (2006). Abundance and diversity of rodents at the human-wildlife interface in Western Serengeti, Tanzania. Afr. J. Ecol., 44, 371-378.

34. Makundi, R. H., Massawe, A. W., Mulungu, L. S., \& Katakweba, A. (2009). Species diversity and population dynamics of rodents in a farm-fallow field mosaic system in Central Tanzania. African Journal of Ecology, 48: 313-320.

35. Men, X. Y., Guo, X. G., Dong, W. G., Ding, N., \& Qian, T. J. (2015). Influence of Human Disturbance to the Small Mammal Communities in the Forests. Open Journal of Forestry, 5: 1-9.

36. Mistrot, V. (2000). Les micromammifères, marqueurs de l'anthropisation du milieu. Études rurales, 9 p.

37. Rosevear, D. R. (1969). The rodents of West Africa. Trustees of the British Museum (Natural History), London, 604 p.

38. Schwitzer, C., Glatt, L., Nekaris, K. \& Ganzhorn, J. U. (2011). Responses of animals to habitat alteration: an overview focusing on primates. Endangered species research, 14: 31-38.

39. Shannon C.E. 1948. A Mathematical Theory of Communication. Bell System Technical Journal, 27(3): 379-423.

40. Stork, N. E., Coddington, J. A., Colwell, R. K., Chazdon, R. L., Dick, C. W., Peres, C. A., Sloan, S., \& Kathy, W. K. (2009). Vulnerability and Resilience of Tropical Forest Species to Land-Use Change. Conservation Biology, 23 (6): 1438-1447.

41. Wilson, D. E., Cole, F. R., Nichols, J. D., Rudran, R., \& Foster, M. S. (1996). Measuring and monitoring biological diversity. Standard methods for mammals. Smithsonian Institution Press, Washington: 409 p. 